\title{
Accuracy Estimation and Usage Experience of the Thermal Anomalies Detecting Algorithms Based on Himawari-8 Satellite Data
}

\author{
Ivan V. Balashov ${ }^{l}$, Mikhail A. Burtsev ${ }^{l}$, Alexey A. Mazurov ${ }^{l}$, Konstantin S. Senko ${ }^{l}$, \\ Oleg V. Gutsalov', Ilya S. Pustinskiy', Alexey O. Chudin', Vladislav D. Yan ${ }^{2}$ \\ ${ }^{1}$ Space Research Institute, Russian Academy of Sciences, Moscow, Russia \\ ivbalashov@d902.iki.rssi.ru \\ ${ }^{2}$ Far Eastern Center of State Research Center "Planeta", Khabarovsk, Russia \\ pis@dvrcpod.ru
}

\begin{abstract}
Temperature anomalies detection using satellite data is one of the traditional tasks now. In addition to MODIS and VIIRS instruments products based on data from Aqua, Terra, Suomi NPP, JPSS NOAA-20 satellites widely used for fire monitoring, products obtained from the AHI instrument of the Japanese geostationary satellite Himawari-8 became available since October 2014. Himawari-8 satellite provides an unprecedented combination of the frequency of observations and the spatial resolution of the received data. This allowed new algorithms for detecting temperature anomalies based not only on the analysis of the temperature and spatial characteristics of fires, but also their temporal dynamics. Such an algorithm, in particular, was developed by the specialists of the Far Eastern Center of SRC "Planeta". This article is devoted to the analysis of the accuracy and efficiency of this algorithm.
\end{abstract}

Keywords: Himawari-8, satellite monitoring, fire monitoring, satellite data processing, AHI, MODIS, VIIRS. 


\title{
ОЦЕНКА ТОЧНОСТИ И ОПЫТ ИСПОЛЬЗОВАНИЯ АЛГОРИТМОВ ДЕТЕКТИРОВАНИЯ ТЕРМИЧЕСКИХ АНОМАЛИЙ ПО ДАННЫМ СПУТНИКА HIMAWARI-8
}

\author{
И.В. Балашов ${ }^{1}$, М.А. Буриев ${ }^{1}$, А,А. Мазуров ${ }^{1}$, К.С. Сенько ${ }^{1}$, \\ О.В. Гуиалов ${ }^{2}$, И.С. Пустынский ${ }^{2}$, А.О. Чудин ${ }^{2}$, В.Д. Ян ${ }^{2}$ \\ ${ }^{1}$ Институт космических исследований РАН, Москва, Россия \\ ivbalashov@d902.iki.rssi.ru \\ ${ }^{2}$ ДЦ ФГБУ «НИЦ «Планета», Хабаровск, Россия \\ pis@dvrcpod.ru
}

\begin{abstract}
Выявление температурных аномалий по спутниковым данным в настоящее время является одной достаточно традиционных задач. В дополнение к широко используемым для мониторинга пожаров продуктам по данным прибора MODIS и VIIRS аппаратов Aqua, Terra, Suomi NPP, JPSS NOAA-20 с октября 2014 года стали доступны продукты, получаемые по данным прибора AHI японского геостационарного аппарата Himawari-8. Данный аппарат обеспечивает беспрецедентную совокупность частоты наблюдений и пространственного разрешения получаемых данных. Это позволило создать новые алгоритмы детектирования температурных аномалий, основанных на анализе не только температурных и пространственных характеристик аномалий, но и их временной динамики. Такой алгоритм, в частности, был создан специалистами ДЦ НИЦ «Планета». Настоящая статья посвящена анализу точности и эффективности работы данного алгоритма.
\end{abstract}

Ключевые слова: Himawari-8, спутниковый мониторинг, мониторинг пожаров, обработка спутниковых данных, AHI, MODIS, VIIRS.

\section{Введение}

Выявление пожаров по спутниковым данным является одной из традиционных и востребованных задач в современных системах дистанционного мониторинга. Сегодня в таких системах широко используются результаты работы алгоритмов, выявляющих температурные аномалии по данным аппаратов AQUA, TERRA, Suomi NPP, Sentinel-2, Метеор-M, аппаратов серий NOAA и Landsat.

В октябре 2014 года был выведен на орбиту японский метеорологический аппарат Himawari-8. Данные, получаемые с установленного на нем прибора АНI, отличаются беспрецедентным для геостационарных аппаратов сочетанием разрешения и частоты наблюдений. Эти характеристики обеспечивают, в частности, возможность получать изображение Земли каждые 10 минут. Такая частота наблюдений позволила специалистам ДЦ НИЦ «Планета» создать алгоритм детектирования температурных аномалий, использующий кроме традиционных температурных и пространственных параметров дополнительный временной контекст [1]. 
Для использования данных о «горячих точках», получаемых в результате работы созданного алгоритма, в существующих информационных системах крайне важен вопрос интерпретации их характеристик. Одним из наиболее применимых на практике способов оценки характеристик получаемой информации о температурных аномалиях является их сравнительная оценка с данными достаточно давно используемых алгоритмов, работающих по данным MODIS и VIIRS [2]. Кроме спутниковых данных для сравнительной оценки возможно использование сообщений о пожарах от наземных служб и информации о выявленных повреждениях растительности на пройденных огнем площадях (гарях).

Решаемой в настоящей работе задачей является сравнение информации о температурных аномалиях, получаемых по данным прибора АНІ алгоритмом, созданным специалистами ДЦ НИЦ «Планета» [1] (далее по данным АНІ), с имеющимися данными о пожарах и гарях с целью получения сравнительных характеристик. В работе приводится описание используемой информации о горении, пожарах и повреждениях растительности, основные особенности алгоритмов их получения. Предлагается набор сравнительных характеристик и методика для их получения. Приводятся результаты, полученные по описанной методике, и формулируются основные выводы.

\section{Исходные данные для сравнительной верификации}

В качестве источников опорных данных в работе использовалась БД пожаров, накопленная в ИКИ РАН на основе информации о детектировании активного горения (горячих точек) по данным приборов VIIRS и MODIS [3]. Основные характеристики этих приборов и особенности алгоритмов обработки получаемых с них данных, а также сведения о Himawari-8 и алгоритме обработки данных AНI кратко будут описаны далее.

Прибор MODIS (Moderate resolution Imaging Spectroradiometer - сканирующий спектрорадиометр среднего разрешения) [4], установленный на аппараты Aqua и Terra, обеспечивает съемку земной поверхности в 36 спектральных каналах с разрешением от 250 до 1000 метров на пиксель. Каждая точка поверхности Земли наблюдается с аппаратов Aqua и Terra до четырех раз в день. Температурные аномалии («горячие точки») по данным прибора MODIS в настоящее время вычисляются порогово-контекстуальным алгоритмом MOD14 [5]. К рассмотрению принимаются только точки, у которых радиояркостная температура в канале 3.5-4 мкм превышает порог в 305 К в ночное время и 310 K в дневное время. Точки с температурой выше верхнего порога 360 К считаются заведомо пожарами. В диапазоне значений между этими порогами работает контекстуальный алгоритм, проводящий маскирование и статистический анализ температурных полей в различных каналах прибора MODIS в окрестности исследуемой точки. Итоговое разрешение пикселя «горячей точки» по данным MODIS составляет 1 км, однако фактическая площадь детектированного горения может быть в несколько раз меньше и в системах, разработанных в ИКИ РАН, определяется специальным коэффициентом коррекции, сформированным на основе статистических данных о повреждениях растительности [6]. Для получения данных о «горячих точках» по исходным спутниковым данным в системах ИКИ РАН применяется программный комплекс NASA IPOPP [7]. В частности, комплекс используется для обработки данных сеансов, получаемых в центрах ФГБУ НИЦ Планета [8], и данных центра NASA LANCE (NRT, близко к реальному времени). Кроме результатов обработки исходных данных используется также готовый продукт температурных аномалий из центра FIRMS NASA [9].

Прибор VIIRS (Visible Infrared Imaging Radiometer Suite) [10], установленный на борту аппаратов Suomi NPP и NOAA 20, имеет 22 спектральных канала с пространственным разрешением от 375 метров на пиксель (I-bands) до 750 метров (M-bands). Suomi NPP 
имеет близкие с Аqua орбитальные параметры и обеспечивает съемку каждой точки земной поверхности до двух раз в сутки. Алгоритм выделения температурных аномалий по данным VIIRS основан на алгоритме MOD14 и использует данные каналов 3,7 и 11 мкм как основные. В системах ИКИ РАН используюся данные, получаемые программным комплексом IPOPР на основе принятых в центрах ФГБУ НИЦ Планета сеансов, а также продукт FIRMS с разрешением 375 метров.

В системах спутникового мониторинга пожаров, создаваемых в ИКИ РАН, данные о «горячих точках», в том числе для оценки пройденной огнем площади, агрегируются как по пространству, так и по времени. Для этого реализована специальная технология ведения базы данных контуров пожаров [10]. Технология основана на том, что по мере поступления новых данных наблюдений по району действия пожара они добавляются к ранее сформированному состоянию пожара. Таким образом, «горячие точки», полученные по отдельным наблюдениям пожара, формируют непрерывный объект - пожар, имеющий интервал действия и область горения. Для задач сравнительной верификации данных Himawari-8 использовались контура объединенных пожаров по данным MODIS и VIIRS (далее, просто MODIS).

Геостационарный аппарат Himawari-8 имеет на борту прибор AHI (Advanced Himawari Imager) [11] с 16-ю спектральными каналами от 0.47 до 13.3 мкм с разрешением от 500 до 2000 метров. В отличие от низкоорбитальных аппаратов, Himawari предоставляет наблюдения каждой точки в области видимости аппарата каждые 10 минут. Для данных прибора AНI KA «Himawari-8» специалистами Дальневосточного центра НИЦ Планета был разработан оригинальный алгоритм и программное обеспечение для детектирования точек вероятного возгорания. В алгоритме для выявления «горячих точек» помимо традиционных методов детектирования используется метод, основанный на главном преимуществе геостационарных спутников - высокой частоте съемки одной и той же территории. В данном методе использован специальный пространственно-временной контекст, позволяющий достичь оптимального баланса между периодичностью наблюдения и вероятностью детектирования мелких очагов горения на ранней стадии [1].

\section{Дополнительные данные для верификации}

Помимо информации о температурных аномалиях, выделяемых по данным различных спутниковых систем, для подтверждения достоверности данных, получаемых по Himawari-8, можно использовать данные о пожарах, полученные от наземных служб МЧС и региональных диспетчерских служб Авиалесоохраны, получаемых в системе ИСДМРослесхоз [12,13]. При совпадении сообщения о пожаре по времени и координатам с данными о температурной аномалии Himawari-8 их можно считать подтвержденными и достоверными с большой долей вероятности.

Принципиально другим источником, способным подтвердить данные о факте горения на поверхности Земли, являются данные о повреждении растительности в результате пожара [14,15]. Гари (пройденные огнем площади), могут быть выявлены по спутниковым данным различного разрешения. При этом во время непосредственно горения из-за наличия облачности или других факторов данных по температурным аномалиям на спутниковых снимках может и не наблюдаться. Факт наличия детектированной гари в области имеющихся «горячих точек» по данным Himawari-8 с большой долей вероятности говорит о том, что в момент съемки действительно наблюдался факт горения.

Отметим, что в информационных системах, создаваемых ИКИ РАН, реализованы две технологии получения контуров пройденных огнем площадей (гарей). Первая ориентирована на данные среднего разрешения прибора MODIS и автоматически формирует контура гарей на всей территории РФ [14]. Вторая предназначена для интерактивного выделения контуров по данным высокого разрешения, таких как данных с 
аппаратов серий Landsat, Sentinel-2 с применением инструментов классификации изображений [16].

\section{Информация для проведения верификации}

Для проведения верификации были использованы данные о пожарах MODIS и VIIRS за сезон 2017 года (данные получены, в частности, из Центра коллективного пользования «ИКИ-Мониторинг» [17], обеспечивающего работу сервисов ВЕГА [18,19]) на территорию Приморского края, имеющего полное покрытие данными Himawari-8. На рассматриваемый период также были использованы сообщения о пожарах от регионального отделения НЦУКС (МЧС), полученные из системы Вега-Приморье [20], и сведения о пожарах, предоставлямые региональной диспетчерской службой ФГБУ «Авиалесоохрана». Кроме этого были использованы данные по повреждениям растительности (гари), полученные по данным MODIS.

\section{Методика сравнительной верификации}

Методика сравнительной верификации основана на подтверждении информации о горении по «горячим точкам» Himawari-8 данными других источников.

Сопоставляя данные о «горячих точках» Himawari-8 с другими данными, можно определить, в частности, какой процент «горячих точек» является подтвержденным, т.е. достоверным, и какой процент пропусков имеет источних данных АНІ относительно них. Сравнение с термальными аномалиями, получаемыми на основе обработки оперативных данных спутниковых полярно-орбитальных систем, может также ответить на вопрос, насколько раньше прибор АНІ позволяет детектировать пожары, какие минимальные площади горения он способен зарегистрировать. Наблюдения «горячих точек» по данным AHI, не подтвержденные информацией о пожарах по данным MODIS и VIIRS, могут быть подтверждены сведениями о повреждениях растительного покрова, выявленных по данным различного разрешения в течение или в конце пожароопасного сезона, и дополнить процент достоверных данных.

Для несопоставленных «горячим точкам» по данным Himawari-8 пожаров MODIS будут получены их характеристики - площади, время действия и температуры первого наблюдения. Распределения значений этих характеристик необходимы для оценки того, какие именно пожары MODIS пропускает прибор AHI.

Для сопоставления с данными MODIS и VIIRS были использованы контура объединенных в пожары точек детектированного активного горения. Для точечных данных наземных служб и МЧС дополнительно строился пространственный буфер - окружность заданного радиуса. Для верификации по повреждениям растительности использовались контура полигонов повреждений, построенных по спутниковым данным высокого и среднего разрешения приборов MODIS, ETM+, OLI-TIRS/OLI, MSI.

Таким образом, в результате проведенного анализа были получены следующие характеристики для оценки работы анализируемого алгоритма выявления температурных аномалий по данным АНI:

- оценка количества пропусков наблюдений горения и ложных срабатываний;

- способность алгоритма детектировать объекты небольших размеров и низких температур: площади сопоставленных пожаров по MODIS и VIIRS и их температуры;

- возможность раннего детектирования пожаров: статистики по времени регистрации сопоставленных пожаров по данным VIIRS и MODIS.

Алгоритм верификации и полученные результаты 
Алгоритм верификации ориентирован на работу со структурой специализированных БД пожаров и данных других источников, используемых в ИКИ РАН. На первом этапе для каждой из «горячих точек» Himawari-8 производится поиск соответствующих во времени и пространстве объектов: пожаров MODIS и VIIRS, гарей, данных субъекта и т.д. Далее полученная информация была сгруппирована по типам сопоставленных данных и получены статистики по их параметрам. На этом этапе также была проверена гипотеза о влиянии количества повторов наблюдения Himawari-8 в одной точке на их достоверность. Для несопоставленных с другими источниками «горячих точек» также были вычислены статистики по распределениям температур, времени регистрации и количеству повторов наблюдения в точке.

Проведенное сопоставление информации о температурных аномалиях, полученных разработанным в ДЦ «НИЦ «Планета» алгоритмом, с другими источниками данных показало в целом достаточно высокий процент подвеждения.

Для оценки влияния количества повторов наблюдений в одной точке были дополнительно оценены три набора «горячих точек» Himawari-8: с только одним наблюдением в точке в течение получаса, набор данных с количеством точек в сериях наблюдений от 2 до 5, и набор данных с сериями, имеющими более 5 наблюденияй. Серией считался набор «горячих точек», наблюдаемых последовательно с промежутком между наблюдениями не более получаса.

Статистика подтверждаемости «горячих точек» по трем наборам данных приведена на рисунке 1 для разных типов сопоставляемой информации.

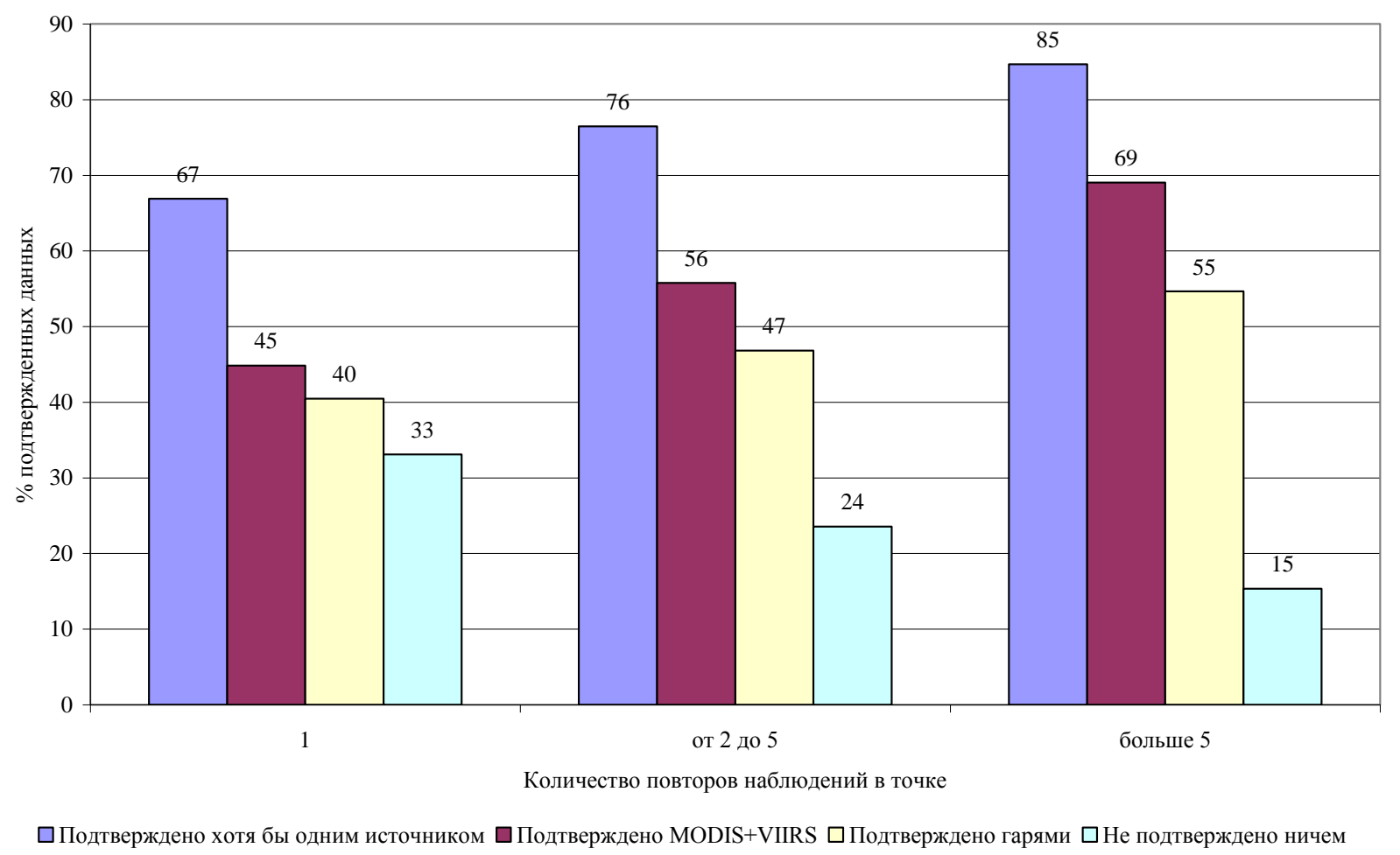

Рис. 1. Статистика подтверждения данных Нітажагі различными источниками в процентах

Полученная статистика показывает наличие положительной зависимости подтверждаемости данных от количества повторов наблюдения, Следует отметить, что данные без фильтрации по количеству повторов наблюдений обладают достаточно высоким процентом подтвержденности (75\%) «горячих точек», детектированных на основе анализа данных Himawari-8. Этот факт говорит о хорошем механизме фильтрации шумов и ложных срабатываний в алгоритме выявления температурных аномалий [1]. Дальнейшая сравнительная верификация проводилась именно для этого набора данных. 
Важным показателем сопоставления является соотношение подтвержденных и пропущенных пожаров по данным MODIS и VIIRS. Статистика показывает, что в сезоне 2017 года AHI «пропустил» всего 23\% пожаров по данным более высокого пространственного разрешения MODIS и VIIRS. Дальнейший анализ подтвержденных данных АНІ пожаров показал, что:

- начиная с площади пожара в 100 гектар, результаты детектирования, полученные на основе данных AHI, практически в $100 \%$ случаев подтверждены данными, детектированными MODIS и VIIRS;

- со 100\% вероятностью алгоритм позволяет детектировать пожары MODIS и VIIRS, имеющие температуру первого наблюдения 350 градусов Кельвина.

Анализ пропущенных AHI пожаров (которые были детектированы на основе данных MODIS или VIIRS) показывает, что данные AHI пропускают в основном пожары, которые действуют менее суток.

Проведение сравнительной оценки времени детектирования пожаров детектированных по данным MODIS или VIIRS c «горячими точками» по данным AHI показало, что информация, поступающая со спутника Himawari-8, позволяет обнаруживать пожары раньше, чем по данным, поступающим с аппаратов AQUA, TERRA, Suomi NPP и NOAA 20 в 64\% случаев. Более детальное рассмотрение показывает, что основная доля пожаров MODIS и VIIRS детектируется данными AHI примерно в то же время (в интервале [10..10] минут). Однако почти $30 \%$ пожаров обнаружено спутником Himawari-8 значительно раньше, а 19\% пожаров детектировались в предыдущих сутках.

\section{Заключение}

Несмотря на низкое пространственное разрешение прибора АНІ КА Himawari-8, алгоритм, созданный специалистами ДЦ НИЦ «Планета», позволяет детектировать очаги горения с характеристиками, сопоставимыми с пожарами по данным MODIS и VIIRS. При этом данные AHI позволяют детектировать горение заметно раньше, чем MODIS, благодаря высокой частоте наблюдений. Пропуски детектирования данными AHI пожаров, детектированных по MODIS и VIIRS, наблюдаются в основном для небольших, низкотемпературных, короткоживущих пожаров, что, естественно, объясняется более низким разрешением прибора AHI по сравнению с приборами MODIS и VIIRS.

Выполненный анализ позволяет сделать вывод о достаточно высокой надежности работы алгоритма детектирования точек вероятного возгорания по данным прибора АНI КА «Himawari-8», разработанного в Дальневосточном центре «НИЦ «Планета» [1].

Проведенные в работе анализ и оценка работы алгоритма детектирования выполнены с использованием ЦКП «ИКИ-Мониторинг» [17].

Работа подготовлена в рамках государственного контракта Министерства науки и высшего образования Российской Федерации №05.577.21.0294 на тему: «Разработка технологий автоматизированной обработки спутниковых данных дистанционного зондирования Земли для создания и поддержки информационных сервисов мониторинга лесных ресурсов и охотничьих угодий России». Уникальный идентификатор проекта RFMEFI57718X0294.

\section{References}

[1] Pustynskiy I.S., J.A. Shamilova, E. I. Kholodov, V.V. Suhanova. The algorithm for detecting hot points from the data of the AHI device Himawari-8, Sovremennye Problemy Distantsionnogo Zondirovaniya Zemli iz Kosmosa, 2018, Vol. 15. No 5, pp. 33-43. (In Russian). 
[2] Loupian E.A., Ershov D.V., Bartalev S.A., Isaev A.S. Information System for Remote Monitoring of Forest Fires and Their Consequences: The Results of the Last Decade and Further Prospects, Aerospace Method and GIS-Technologies in Forestry and Forest Management: Proceedings of the V All-Russian Conference, Dedicated to the Memory of V.Iv. Sukhikh and G.N. Korovin. Moscow, Russia, April 22-24, 2013.- M. CEPL RAS, 2013. P. 40-43. (In Russian).

[3] Flitman E.V., Balashov I.V., Bourtsev M.A., Galeev A.A., Egorov V.A., Kotelnikov R.V., Loupian E.A., Mazurov A.A., Matveev A.M., Proshin A.A. Organization of the MODIS Instrument Data Processing System for the Tasks of Monitoring Forest Fires and Their Aftereffects, Sovremennye Problemy Distantsionnogo Zondirovaniya Zemli iz Kosmosa, 2011, Vol. 8, No 1, pp. 127-138. (In Russian).

[4] URL: https://modis.gsfc.nasa.gov/about/

[5] URL: https://lpdaac.usgs.gov/sites/default/files/public/product_documentation/mod14_atbd.pdf

[6] Stytsenko F.V., Loupian E.A., Bartalev S.A., Ershov D.V. Methods for estimating the area covered by forest fires based on satellite data of different spatial resolution, Sovremennye Problemy Distantsionnogo Zondirovaniya Zemli iz Kosmosa. Moscow, Russia, November 14-18 2016. T. C. 396. (In Russian).

[7] URL: https://directreadout.sci.gsfc.nasa.gov/?id=dspcontent\&cid=68

[8] Bourtsev M.A., Milekhin O.E., Kramareva L.S., Antonov V.N., Balashov I.V., Kashnitskiy A.V., Loupian E.A., Matveev A.M., Proshin A.A., Uspensky S.A. Unified system of distributed work with the data of reception centers "SIC" Planeta ": current opportunities and development prospects, Sovremennye Problemy Distantsionnogo Zondirovaniya Zemli iz Kosmosa. Moscow, Russia, November 12-16 2018, pp. 4. DOI: 10.21046/2070-16DZZconf-2018a. (In Russian).

[9] URL: https://firms.modaps.eosdis.nasa.gov/

[10] URL: https://ladsweb.modaps.eosdis.nasa.gov/missions-and-measurements/viirs/

[11] URL: https://www.data.jma.go.jp/mscweb/en/himawari89/space_segment/spsg_ahi.html

[12] Loupian E.A., Bartalev S.A., Ershov D.V., Kotelnikov R.V., Balashov I.V., Bourtsev M.A., Egorov V.A., Efremov V. Yu., Zharko V.O., Kovganenko K.A., Kolbudaev P.A., Krasheninnikova Yu. S., Proshin A.A., Mazurov A.A., Uvarov I.A., Stytsenko F.V., Sychugov I.G., Flitman E.V., Khvostikov S.A., Shulyak P.P. Satellite data processing management in Forest Fires Remote Monitoring Information System (ISDM-Rosleskhoz) of the Federal Agency for Forestry, Sovremennye Problemy Distantsionnogo Zondirovaniya Zemli iz Kosmosa, 2015, Vol.12, No 5. pp. 222-250. (In Russian).

[13] Loupian E.A., Bartalev S.A., Ershov D.V. Satellite Data Aplication Management in the ISDMRosleshoz System // Aerospace Methods and GIS-Technologies in Forestry, Forest Management and Ecology: Proceedings of the VI All-Russian Conference, Moscow, Russia, April 20-22, 2016. - M. CEPF RAS, 2016. P. 39-42. (In Russian).

[14] Kashnitskiy A.V., Bartalev S.S., Efremov V. Yu., Stytsenko F.V. Burned Area Mapping within Remote Wildfire Monitoring Information Systems Based on High Resolution Satellite Data, Aerospace Methods and GIS-Technologies in Forestry, Forest Management and Ecology: Proceedings of the VI AllRussian Conference, Moscow, Russia, April 20-22, 2016. - M. CEPF RAS, 2016. P. 177-181. (In Russian).

[15] Stytsenko F.V., Bartalev S.A., Ivanova A.A., Loupian E.A., Sychugov I.G. Forest burnt area assessment possibilities in regions of Russia based on active fires detection by satellites, Sovremennye 
Problemy Distantsionnogo Zondirovaniya Zemli iz Kosmosa, 2016, Vol. 13, No. 6, pp. 189-298. DOI: 10.21046/2070-7401-2016-13-6-289-298. (In Russian).

[16] Kashnitskiy A.V., Efremov V.Yu., Loupian E.A., Bartalev S.S. Organization of a mapping system for areas traversed by natural fires using automatic classification procedures, Sovremennye Problemy Distantsionnogo Zondirovaniya Zemli iz Kosmosa. 2015, November 16-20. Abstracts C. 403. (In Russian).

[17] E.A. Loupian, A.A. Proshin, M.A. Burtsev, I.V. Balashov, S.A. Bartalev, V.Yu. Efremov, A.V. Kashnitskiy, A.A. Mazurov, A.M. Matveev, O.A. Sudneva, I.G. Sychugov, V.A. Tolpin, I.A. Uvarov. IKI center for collective use of satellite data archiving, processing and analysis systems aimed at solving the problems of environmental study and monitoring, Sovremennye problemy distantsionnogo zondirovaniya Zemli iz kosmosa, 2015, Vol.12, No 5, pp. 263-284. (In Russian).

[18] Bartalev S.A., Ershov D.V., Loupian E.A., Tolpin V.A., Vozmozhnosti ispol'zovaniya sputnikovogo servisa VEGA dlya resheniya razlichnykh zadach monitoringa nazemnykh ekosistem (Possibilities of Satellite Service VEGA Using for Different Tasks of Land Ecosystems Monitoring ), Sovremennye problemy distantsionnogo zondirovaniya Zemli iz kosmosa, 2012, Vol. 9, No. 1, pp. 49-56. (In Russian).

[19] URL: http://sozvezdie-vega.ru

[20] Loupian E.A., Bartalev S.A., Balashov I.V., Bartalev S.S., Bourtsev M.A., Egorov V.A., Efremov V. Yu., Zharko V.O., Kashnitskiy A.V., Kolbudaev P.A., Kramareva L.S., Mazurov A.A., Oksyukevich A.Y., Plotnikov D.E., Proshin A.A., Senko K.S., Uvarov I.A., Khvostikov S.A., Khovratovich T.S. VegaPrimorie: complex remote forest monitoring information system, Sovremennye Problemy Distantsionnogo Zondirovaniya Zemli iz kosmosa, 2016, Vol. 13, No. 5, pp. 11-28. DOI: 10.21046/20707401-2016-13-5-11-28. (In Russian). 